\title{
Enhanced Hopfield Network for Pattern Satisfiability Optimization
}

\author{
Mohd. Asyraf Mansor \\ School of Mathematical Sciences, Universiti Sains Malaysia, 11800, Pulau Pinang, Malaysia \\ E-mail: asyrafalvez@live.com \\ Mohd Share duwan M. Kasihmuddin \\ School of Mathematical Sciences, Universiti Sains Malaysia, 11800 USM, Penang Malaysia \\ E-mail: iwanmaidin@gmail.com \\ Saratha Sathasivam \\ School of Mathematical Sciences, Universiti Sains Malaysia, 11800 USM, Penang Malaysia \\ E-mail: saratha@usm.my
}

\begin{abstract}
Highly-interconnected Hopfield network with Content Addressable Memory (CAM) are shown to be extremely effective in constraint optimization problem. The emergent of the Hopfield network has producing a prolific amount of research. Recently, 3 Satisfiability (3SAT) has becoming a tool to represent a variety combinatorial problems. Incorporated with 3-SAT, Hopfield neural network (HNN-3SAT) can be used to optimize pattern satisfiability (Pattern-SAT) problem. Hence, we proposed the HNN-3SAT with Hyperbolic Tangent activation function and the conventional McCulloch-Pitts function. The aim of this study is to investigate the accuracy of the pattern generated by our proposed algorithms. Microsoft Visual C++ 2013 is used as a platform for training, testing and validating our Pattern-SAT design. The detailed performance of HNN3SAT of our proposed algorithms in doing Pattern-SAT will be discussed based on global pattern-SAT and running time. The result obtained from the simulation demonstrate the effectiveness of HNN-3SAT in doing Pattern-SAT.
\end{abstract}

Index Terms-Pattern-SAT, Hopfield Network, 3Satisfiability, Hyperbolic Tangent Activation Function, McCulloch-Pitts Function.

\section{INTRODUCTION}

Satisfiability problem is a paradigmatic constraintsatisfaction conundrum with zillions of applications, such as in pattern reconstruction and circuit configuration [1, 16]. Traditionally, the pattern reconstruction deals with the geometrical field and implemented the conventional technique to verify the pattern via neural network [8, 14]. Technically, we proposed a paradigm to verify the pattern and recall it back after certain training process. So, we combine the benefits of 3-Satisfiability, Logic Programming, Hopfield Neural Network and activation function to verify the pattern satisfiability problem. The
Pattern Satisfiability problem (Pattern-SAT) is a brand new conundrum that inspires researchers to create model verification techniques which help in generating correct pattern. For many practical reasons, it is becoming very difficult to manually recall the stored pattern because it will end up with a large number of fault. Hence, the generated pattern will be disturbed by monstrous amount of noises.

In order to minimize the problem, Hopfield neural network is the perfect network due to the existence of remarkable content addressable memory (CAM) [2, 3]. Hence, recurrent neural network are essentially dynamical systems that feedback signals to themselves [6]. Then, the Hopfield neural network is a simple recurrent network which can work as an efficient associative memory, and it can store certain memories in a manner rather analogous to the brain $[3,11]$. In addition, it is a branch of neural network that as sist the researchers in constructing the model of our brain. Thus, it is very practical to verify the pattern satisfiability problem. Hopfield neural network minimizes Lyapunov energy due to physical spin of the neuron states [15, 27]. The network produced optimal output by minimizing the network energy. Gadi Pinkas [9] and Wan Abdullah [4] defined a bi-directional mapping between logic and energy function of symmetric neural network. To frame their novelty, both methods are applicable to validate the solution obtained are models for a corresponding logic program. The work of Sathasivam [11,28] showed that the optimized recurrent Hopfield network could be possibly used to do logic programming. Furthermore, intelligent technique of defining connection strength of the logic helps the network to overwhelm spurious state [15].

Basically, logic programming can be treated as a problem in combinatorial optimization perspective. [12] Therefore, it can be carried out in a neural network to obtain desired solutions. In this paper, we will consider logic programming in Hopfield network. The conventional paradigm is Wan Abdullah's logic 
programming based on McCulloch-Pitts function [4, 7]. Wan Abdullah proposed a paradigm of doing logic program on a Hopfield network. We extended the work related on the neural-symbolic integration by implementing Hopfield network and activation function in doing logic program. As a result, we proposed the Hyperbolic Tangent activation function as the catalyst of doing logic programming in Hopfield network. Since, the Pattern-SAT is involving 3-Satisfiability problem before a pattern can be activated, our proposed hybrid network can accelerate the process of recalling the correct pattern. More importantly, it will be able to retrieve correct pattern as the complexity of the pattern increases [10].

This paper has been organized as follows. Section I, formally introduces the important concept of Pattern Satisfiability, 3-Satisfiability, Hopfield neural network, logic programming and activation function. In section II, the important theory of 3-satisfiability (3-SAT) problems is discussed briefly. Then, section III covers the building block and algorith m for Pattern-SAT. Meanwhile, section IV presents the neuro-logic parts, including the fundamental concept of Hopfield neural network, logic programming and activation function used in our proposed network. In section $\mathrm{V}$, theory implementation of the networks was discussed briefly. Finally, section VI and VII enclose the experimental results, discussion and conclusion.

\section{SATISFIABILITY (SAT) PROBLEM}

Satisfiability problem is a well-known constraint-based problem in mathematics and computer sciences. Besides, the satis fiability problem has emerging as an optimization problem that can be solved by neural network. In this paper, we limit our analys is until 3-Satisfiability (3-SAT).

\section{A. Boolean Satisfiability Problem}

Boolean Satis fiability (SAT) can be demarcated as the decision problem since the solution will yield either "yes" or "no" response, by implementing the Boolean formula $[12,6]$. Hence, SAT involves definitive task of searching truth assignments that satisfies a Boolean formu la [7]. In other words, satisfiability problem refers to verdict problems based on particular constraints. In this paper, we emphasize 3-Satis fiability (3-SAT) to be embedded in Patter-Satisfiability (Pattern-SAT) problem.

\section{B. 3-Satisfiability Problem}

In this paper, we emphasize a paradigmatic NPcomplete problem namely 3-Satsifiability (3-SAT). Generally speaking, 3-SAT can be defined as a formula in conjunctive normal form where each clause is limited to at most or strictly three literals $[21,22]$. The proble $m$ is an example of non-deterministic problem [12]. In our analysis, the following 3-SAT logic program which consists of 3 clauses and 3 literals will be used. For instance:

$$
P=(A \vee B \vee \bar{C}) \wedge(\bar{A} \vee \bar{B} \vee C) \wedge(\bar{A} \vee B \vee D)
$$

We represent the above $3 \mathrm{CNF}$ (Conjunctive Normal Form) formula with P. Thus, the formula can be in any combination as the nu mber of ato ms can be varied except for the literals that are strictly equal to $3[5,24]$. Hence, it is vital for combinatorial optimization problem. The higher number of literal in each clause will increase the possibilities or chances for a clause to be satisfied [11, 22].

The general formula of 3-SAT for conjunctive normal form (CNF):

$$
P=\wedge_{i=1}^{n} Z_{i}
$$

So, the value of $\mathrm{k}$ denotes the number of satisfiability. In our case, $\mathrm{k}-\mathrm{SAT}$ is 3-SAT.

$$
Z_{i}=\wedge_{j=1}^{k}\left(x_{i j}, y_{i j}, z_{i j}\right), k>3
$$

\section{PATtern SATisfiabiLity}

Pattern satisfiability is a brand new topic, combining the advantages of Boolean satisfiability concept and pattern reconstruction as a single optimization problem. Specifically, the pattern satisfiability (Pattern-SAT) requires a robust network to optimize the solutions. In this paper, the solutions refer to the ability to retrieve the correct pattern without noises.

\section{A. Pattern Satisfiability (Pattern-SAT)}

Pattern satisfiability is a mathematical model enthused by the means of Boolean circuit concept. In this paper, we will translate and construct the pattern according to 3Satisfiability problem. The building block of our PatternSAT is depend on the particular 3-SAT formula. Strictly speaking, Pattern-SAT is a brand new combinatorial problem, where we need to consider the satisfied assignment in order to produce a correct pattern. We introduce the pattern satisfiability problem as PatternSAT. The most imminent fact is the pattern will be stored in Hopfield's brain as a content addressable memory (CAM) [2, 14]. Hence, we co mb ine the advantages of the Hopfield neural network and logic programming in pattern reconstruction according to 3-SAT. In our case, we proposed the 3-SAT Hopfield neural network to verify the correctness of the Pattern-SAT. Since, 3-SAT is NP problem, thus this conundrum can be verified within the polynomial time. So, Pattern-SAT can be concluded as NP problem. Note that Pattern-SAT has exactly $2^{n}$ consistent truth assignments before a pattern be activated, where $n$ is the number of input points or the crucial points.

Modelling a correct pattern can be treated as combinatorial problem. We are required to construct the pattern according to the crucial points. First of all, we need to create a pattern according to the crucial points. We represent the crucial points of the pattern as the clause. Each crucial points, $c_{i}$ are represented by 3 
neurons. In order to produce the pattern, all the crucial points need to be activated. The more the crucial points, the more complex the system that we will train [5]. During the learning process, the constructed pattern will be checked before being stored to CAM. The concept similar to the Boolean circuit that requires all the component to activate before fully functioning [14, 17].

The satisfied pattern will be stored in content addressable memory (CAM) by distributing it among neurons [13]. After the relaxation process, we are going to recall the stored pattern the same way as biological brain works $[26,27]$. Technically, the output is based on the correct pattern being recalled by our proposed hybrid network. Hence, in order to check the effectiveness of our proposed model in retrieving the pattern, we run the simu lation until 100 trials. Hence, our proposed algorithm act as a robust brain model that can be implemented in other NP problem. The indicators are the number of correct retrieved pattern and time taken to recall all the patterns, given the 100 trials.

In our study, we will consider an alphabet pattern. We will increase the number of crucial points in order to check whether the retrieval power improve or not. For example, the pattern consist of the following crucial points $I P=\left\{\mathrm{c}_{1}, c_{2}, \ldots . c_{i}\right\}$. In our study we will consider $i=5,10 \ldots .50$.

\section{B. Implementation of Pattern-SAT}

1) Choose any pattern. For our case, we choose the alphabet pattern.

2) Identify the crucial points in the pattern. We represent crucial points in the form of $I P_{i}$.

3) Calculate the number of $I P_{i}$. Each crucial point represent by clauses $c_{i}$ with 2 neurons.

$$
I P_{i}=C_{i}
$$

4) Train $c_{i}$. We used ES, during learning. The network was trained 1000 iterations.

5) Randomize the state of the neurons.

6) The network relax based relaxation equation proposed by Sathasivam [27]

$$
\frac{d h_{i}}{d t}=R \frac{d h_{i}}{d t}
$$

where $h_{i}$ is the local field and $R$ relaxation rate. The relaxation rate $\mathrm{R}$ reflects how fast the network relaxed. The value of $R$ is an adjustable parameter and can be determined empirically.

7) Retrieve $I P_{i}$ by using Wan Abdullah Method [4].

8) Construct the retrieved $I P_{i}$ and check the correctness of the pattern (global pattern).

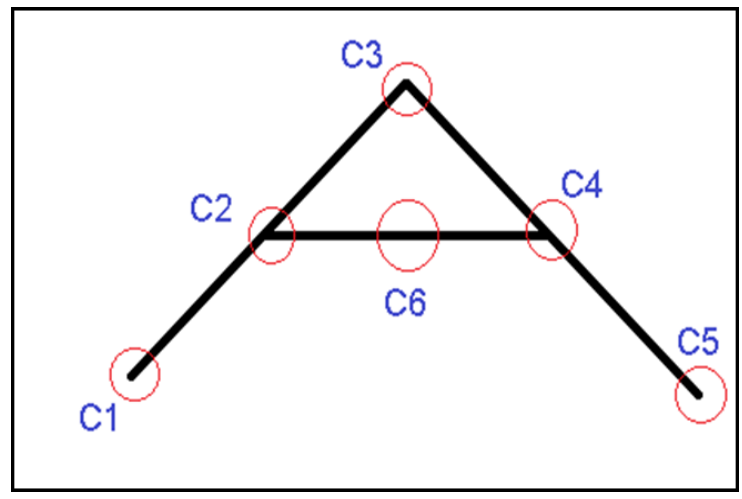

Fig.1. The alphabet used in Pattern-SAT with crucial points.

The above example contains 6 crucial points (circled in red). Each point represents the number of clauses added. Our aim is to ensure all the points are "activated" during retrieval phase. If the clause is satisfied, the points will be activated. The network also required to store the pattern in CAM. The network will be trained 1000 times.

\section{NEURO-LOGIC IN HOPFIELD NEURAL NET WORK}

Neuro-logic depicts the integration of Hopfield neural network and logic programming as a single hybrid network. In this paper, we enhanced the hybrid network by introducing the post optimization techniques, by using Hyperbolic Tangent activation function to solve Pattern satisfiability problem. In order to check the performance, we compared the proposed techniques with the conventional method, McCulloch-Pitts function.

\section{A. Hopfield Model}

In pattern satisfiability, we choose the Hopfield neural network because it is distributed and can be hybridized with the other algorithm [20, 21]. The pattern reconstruction is based on the crucial points and the ability to recall the stored pattern. Specifically, we opt to integrate 3-Sat isfiability and the Hopfield neural network as HNN-3SAT in order to solve the pattern satisfiability (Pattern-SAT) problem [24, 25]. Basically, the Hopfield neural network is an ordinary model for content addressable me mory (CAM). This structures emulates our biological brain whereby learning and retrieving data are the building block of content addressable me mo ry (CAM) $[2,3]$. The main concern is the ability of our proposed model to recall the correct pattern from the CAM.

In this paper, we will consider Discrete Hopfield Neural Network. Discrete Hopfield neural network is a class of recurrent auto-as sociative network $[11,15]$. The units in Hopfield models are mostly binary threshold unit. Hence, the Hopfield nets will take a binary value such as 1 and -1 .

The demarcation for unit I's activation, $a_{i}$ are given as follows:

$$
a_{i}=\left\{\begin{array}{l}
1 \quad \text { if } \sum_{j} W_{i j} S_{j}>\xi_{i} \\
-1 \text { Otherwise }
\end{array}\right.
$$


Where $W_{i j}$ is the connection strength from unit $j$ to $i$. $S_{j}$ is the state of unit $j$ and $\xi_{i}$ is the threshold of unit $i$. The connection in Hopfield net typically has no connection with itself $W_{i j}=0$ and weight connections are symmetric or bidirectional.

Hopfield network work asynchronously with each neuron updating their state determin istically. The system consists of $N$ formal neurons, each is described by an Ising variable. Neurons are bipolar $S_{i} \in\{1,-1\}$ obeying the dynamics $S_{i} \rightarrow \operatorname{sgn}\left(h_{i}\right)$ where the local field $h_{i}$ [6, 27]. The connection model can be generalized to include higher order connection. This modifies the field to

$$
h_{i}=\sum_{j} W_{i j}^{(2)} S_{j}+J_{i}^{(1)}
$$

The weight in Hopfield network is constantly symmetrical. The weight in Hopfield network refers to the connection strength between the neurons [4, 11]. The updating rule maintains as follows:

$$
S_{i}(t+1)=\operatorname{sgn}\left[h_{i}(t)\right]
$$

This property guarantee the energy will decrease monotonically even though following the activation system [24, 28]. Hence, it will drive the network to hunt for the minimum energy. The following equation represents energy for Hopfield network.

$$
E=\ldots-\frac{1}{2} \sum_{i} \sum_{j} W_{i j}^{(2)} S_{i} S_{j}-\sum_{i} W_{i}^{(1)} S_{j}
$$

\section{B. Logic Programming in Hopfield Network}

Logic programming is user writable, readable and be carried out in a neural network to obtain desired solutions.

This can be done by using the neurons to store the truth values of the literal and writing a cost function which is minimized when all clauses are satis fied $[12,19]$. In other words, the main task is to find 'models' corresponding to the given logic program. The fundamental of Hopfield network in doing logic programming was brought up because of its unique content addressable memory properties.

Implementation of HNN-3SAT for Pattern-SAT in Logic Programming.

i. The 3-SAT clauses are translated and transformed into Boolean algebra. Basically, the clauses will form a formula that will decide the overall satisfiability. In Pattern-SAT, the clauses are the combination of crucial points in that particular pattern.

ii. Identify a neuron to each ground neuron.

iii. Initialize all connection strengths or weights to zero. iv. Derive a cost function that is related with negation of all 3-SAT clauses. For instance,
$X=\frac{1}{2}\left(1+S_{X}\right)$ and $\bar{X}=\frac{1}{2}\left(1-S_{X}\right) . S_{X}=1$ (True) and $S_{X}=-1$ (False). Multiplication represents CNF and addition represents DNF.

v. Comparing the cost function with energy, $E$ by obtaining the values of the connection strengths. (Sathasivam's Method) [2, 27]

vi. Check clauses satisfaction by using exhaustive search. Hence, the satisfied clauses will be stored. In Pattern-SAT, the satisfied patterns will be stored as content address able memory.

vii. The states of the neurons are randomized. The network undergoes sequences of network relaxation. Compute the corresponding local field $h_{i}(t)$ of the state. If the final state is stable for 5 runs, we ponder it as final state.

viii. Find the corresponding final energy $E$ of the final state by using Lypunov equation (9). Validate whether the final energy obtained is a global minimum energy or local minima. In Pattern-SAT, the final energy depicts the number of Global Pattern-SAT generated after each simu lation. Next, the CPU time shows the performance of the proposed network to recall the correct pattern.

\section{McCulloch-Pitts Function}

The McCulloch-Pitts model of a neuron is modest yet has considerable computing prospective. The McCullochPitts function is also having precise mathematical definition and easy to implement. Conversely, this model is very simplistic and tends to generate local minima outputs $[11,16]$. Many researchers urged the alternatives for this function as it is very primitive and leads to slower convergence.

$$
\mathrm{g}(x)=x
$$

Another drawback is the linear activation function will only produce positive numbers over the whole real number. Thus, the output values are unbounded and not monotonic [3]. Hence, the outputs obtained will diverges from the global solutions.

\section{Hyperbolic Tangent Activation Function}

Hyperbolic tangent activation function is proven as the most commanding and robust activation function in neural network $[11,15]$. Therefore, we want to explore whether the robustness of this function will apply to the 3-SAT logic programming in Pattern-SAT. Commonly, this function can be defined as the ratio between the hyperbolic sine and the cosine functions expanded as the ratio of half-difference and half-sum of two exponential functions in the points $\mathrm{x}$ and $-\mathrm{x}$ [10]. The Hyperbolic tangent activation function is written as follows:

$$
g(x)=\tanh (x)=\frac{\sinh (x)}{\cosh (x)}=\frac{e^{x}-e^{-x}}{e^{x}+e^{-x}}
$$

The main feature of Hyperbolic tangent activation 
function is the broader output space than the linear activation function. The output range is -1 to 1 , similar to the conventional sigmoid function $[6,10]$. The scaled output or bounded properties will assist the network to produce good outputs. Hence, the outputs will converge to the global solutions.

\section{IMPLEMENT ATION}

For implementation, we assigned any pattern for the network. The crucial points of the pattern will be determined. Each crucial points will generate random program 3-SAT clauses. From there, we in itialized initial states for the neurons in the clauses. The network will evolve until final state reached. Once the program has reached the final state, the neuron state is updated via equation (7). As soon as the network relaxed to an equilibrium state, test the final state obtained for the relaxed neuron whether it is a stable state. Stable state will be considered provided the state remains unchanged for five runs. According to Pinkas [9], letting an ANN to evolve that lead to stable state where the energy function obtained does not change further. In this case, the corresponding final energy for the stable state will be calculated. If the difference between the final energy and the global minimum energy is within the given tolerance value, then consider the solution as global pattern.

\section{RESULTS AND DISCUSSION}

In this paper, Microsoft Visual C++ 2013 was used in simulating the results. In order to validate our proposed network, we carried out the simu lations by using different number of crucial points. The main aspect in our analys is are the global Pattern-SAT and the CPU time for the enhanced network in pattern satisfiability optimization.

\section{A. Global Pattern-SAT}

To begin with, global Pattern-SAT is the percentage of correct pattern retrieved after 100 trials. The term ' $\mathrm{g}$ lobal' here depicts the global solutions obtained in the form of correct pattern.

Table 1 elucidates the number of correct pattern retrieved after 100 trials for different number of crucial points. The capability to recall the correct pattern by implementing our proposed paradigm is the core impetus of our research. In this paper, we limit our number of crucial points to 50 as the training part consumes more time as we need to run until 100 trials. After that, we applied the recalling algorithm and undergo a systematic relaxation process. The results demonstrate that, if we increase the number of crucial points to be stored in CAM, a significant decrease in the number of correct pattern generated at the end of the simulations. However, the correct pattern is still with in $85 \%$ to $100 \%$ as the number of crucial points are being increased for every simulation. This implies that our proposed technique is efficient and consistent in retrieving the output after the training process.
Table 1. Global Pattern-SAT

\begin{tabular}{|c|c|c|}
\hline \multirow{2}{*}{$\begin{array}{c}\text { Number of } \\
\text { Crucial } \\
\text { Points }\end{array}$} & $\begin{array}{c}\text { The Global Pattern-SAT (\%) } \\
\text { 35AT With } \\
\text { McCulloch-Pitts } \\
\text { Function) }\end{array}$ & $\begin{array}{c}\text { Pattern-SAT (HNN- } \\
\text { 3SAT with } \\
\text { Hyperbolic Tangent } \\
\text { Activation Function) }\end{array}$ \\
\hline 5 & 99 & 100 \\
\hline 10 & 99 & 100 \\
\hline 15 & 96 & 100 \\
\hline 20 & 95 & 100 \\
\hline 25 & 92 & 99 \\
\hline 30 & 90 & 99 \\
\hline 35 & 87 & 96 \\
\hline 40 & 85 & 95 \\
\hline 45 & 82 & 95 \\
\hline 50 & 82 & 90 \\
\hline
\end{tabular}

Based on Table 1, Pattern-SAT with Hyperbolic Tangent activation function outperforms Pattern-SAT without any activation function in term of the number of correct pattern retrieved. When the number of neurons increased, Pattern-SAT with Hyperbolic Tangent activation is able to sustain more neurons. Thus, we can clearly observe from the result that the number of correct recalled pattern is consistently 100 until 20 crucial points and maintain $90 \%$ of correctness when the 50 crucial points are used. The limit for our proposed model is until 50 neurons. After 50 neurons, the network is stuck in trial and error state in a long period of time. The whole patterns in exhaustive search method will be inactivated when one of the crucial points is not satisfied. In addition, it might take a longer time for the network to search the correct neuron states and proceed with the relaxation state as the network spends more time in training state. However, the introduction of Hyperbolic Tangent activation function, which is well-known as a robust function by Sathasivam [28], will help the network to relax and be able to recall more patterns correctly. This method will give more time for the network to relax and retrieve the correct states systematically. On the contrary, less relaxation time will create spurious minima which will cause the retrieved solution to achieve local minima and produce wrong pattern. Hence, our proposed technique performs consistently in recalling the stored pattern in CAM under higher complexity and larger circumstances. Thus, the HNN-3SAT WITH Hyperbolic Tangent activation function outperformed $\mathrm{McCu}$ llochPitts function.

\section{B. CPU Time}

In this paper, the CPU time can be defined as the time taken for a logic program to generate and retrieve the correct patterns. Thus, it is an indicator to test the robustness of our proposed algorithm in pattern reconstruction involving 3-Satisfiability cases. Theoretically, our biological brain requires more time to store and retrieve any information if the complexity increases. 
Table 2. CPU Time for the Pattern-SAT

\begin{tabular}{|c|c|c|}
\hline \multirow{2}{*}{\begin{tabular}{c} 
Number of $\begin{array}{c}\text { Crucial } \\
\text { Points }\end{array}$ \\
\cline { 2 - 3 }
\end{tabular}} & $\begin{array}{c}\text { CPU Time } \\
\text { (HNN-3SAT } \\
\text { without Activation } \\
\text { Function) }\end{array}$ & $\begin{array}{c}\text { Pattern-SAT (HNN- } \\
\text { 3AT with Hyperbolic } \\
\text { Tangent Activation } \\
\text { Function) }\end{array}$ \\
\hline 5 & 5 & 2 \\
\hline 10 & 19 & 8 \\
\hline 15 & 230 & 56 \\
\hline 20 & 783 & 122 \\
\hline 25 & 1982 & 456 \\
\hline 30 & 3400 & 723 \\
\hline 35 & 6200 & 1169 \\
\hline 40 & 13488 & 3204 \\
\hline 45 & 25684 & 6422 \\
\hline 50 & 66250 & 10450 \\
\hline
\end{tabular}

Table 2 portrays the CPU time for the Pattern-SAT. According to the results obtained, it can be clearly seen that CPU time increases when the number of crucial points increases. For instance, the complexity of the network increased as the number of crucial points gets massive. We can see that the computational time increased when the nu mber of neuron was getting higher. This is due to the condition when the network was getting larger and complex, the network was probably to get stuck in local minima and devour more computation time. As a result, extra time was needed to relax to global solution as the number of neurons increased. Moreover, the neurons needed to jump enormous energy barrier to reach the global solutions. On separate note, the pattern generation process whereby all the crucial points need to be activated usually consumes more computational time due to the trial and error process in hunting the satisfied interpretation.

The computation time obtained for Pattern-SAT with Hyperbolic Tangent activation function outperformed the McCulloch-Pitts in term of computation time. When we applied the Hyperbolic Tangent activation function to our network, the fixed bound allowed the network to retrieve the correct state much faster. Hence, the correct pattern can be obtained due to less computation burden for each number of crucial points. The traditional paradigm, McCulloch-Pitts function will retrieve the correct states in slower pace due to the complexity and computation burden.

\section{CONCLUSION}

The direct implementation of logic programming in Hopfield network by implementing activation function in verifying the pattern satisfiability had been explored in this research. The number of correct pattern recalled and CPU time indicated that our proposed network, HNN3SAT with Hyperbolic Tangent activation outperformed the HNN-3SAT with McCulloch-Pitts function in
Pattern-SAT. From the theory and experimental results, the proposed network with Hyperbolic Tangent activation function enhanced the chance of retrieving more correct patterns as the complexity is getting higher. The performance analysis is supported by the very good agreement of the global pattern and CPU time obtained.

\section{REFERENCES}

[1] S. Kumar \& M. P. Singh, Pattern recall analysis of the Hopfield network with a genetic algorithm, Computer and Mathematic with Applications, 60, 1049-1057, 2010.

[2] J. J. Hopfield, D. W. Tank, Neural computation of decisions in optimization problem, Biological Cybernatics, 52, 141-152, 1985.

[3] S. Haykin, Neural Networks: A Comprehensive Foundation, New York: Macmillan College Publishing, 1999.

[4] W.A.T. Wan Abdullah, Logic Programming on a Neural Network. Malaysian Journal of computer Science, 9 (1), $1-5,1993$.

[5] T. Larabee, Test pattern generation using Boolean satisfiability, IEEE Transaction on Computer Aided Design, 11(1), 4-15, 1992.

[6] S. Sathasivam, Energy Relaxation for Hopfield Network with the New Learning Rule, International Conference on Power Control and Optimization, 1-5, 2009.

[7] C. Rene \& L. Daniel, Mathematical Logic: Propositional Calculus, Boolean Algebras, Predicate Calculus, United Kingdom: Oxford University Press, 2000.

[8] V. Sivaramakhrisnan, C. S. Sharath, \& P. Agrawal, Parallel test pattern generation using Boolean satisfiability, IEEE Int. Sy mposium on VLSI design, 69-74, 1991.

[9] G. Pinkas, R. Dechter, Improving energy connectionist energy minimization, Journal of Artificial Intelligence Research, 3, 223-15, 1995.

[10] K. Bekir and A. O. Vehbi, Performance analysis of various activation functions in generalized MLP architectures of neural network. International Journal of Artificial Intelligence and Expert Systems 1(4), 111-122, 2010.

[11] M. Velavan, Boltzman Machine and Hyperbolic Activation Function in Higher Order Network, 9 (2), 140 146, 2014.

[12] R.A. Kowalski, Logic for Problem Solving. New York: Elsevier Science Publishing, 1979.

[13] A. Nag, S. Biswas, D. Sarkar, P. P. Sarkar \& B. Gupta, A simple feature extraction technique of a pattern by Hopfield network, International Journal of Advancements in Technology, 45-49, 2000.

[14] C. Ramya, G. Kavitha, \& K. S. Shreedhara, Recalling of images using Hopfield network model, Proceeding for National Conference on Computers, Communication and Control 11, 2011.

[15] S. Sathasivam, P.F. Ng, N. Hamadneh, Developing agent based modelling for reverse analysis method, 6 (22), 4281-4288, 2013.

[16] R. Rojas, Neural Networks: A Systematic Introduction. Berlin: Springer, 1996.

[17] R. Puff, J. Gu, A BDD SAT solver for satisfiability testing: An industrial case study, Annals of Mathematics and Artificial Intelligence, 17 (2), 315-337, 1996.

[18] F. A. Aloul, A. Sagahyroon, Using SAT-Based Techniques in Test Vectors Generation, Journal of Advance in Information Technology, 1 (4), 153-162, 2010.

[19] T. A. Junttila, I. Niemela, Towards an efficient tableau method for Boolean circuit satisfiability checking, in 
Computational Logic-CL 2000, Berlin, Heidelberg: Springer, 553-567, 2000.

[20] A. Cimatti, M. Roveri, Bertoli. P, Conformant planning via symbolic model checking and heuristic search, Artificial Intelligence, 159 (1), 127-206, 2004..

[21] U. Aiman and N. Asrar, Genetic algorithm based solution to SAT-3 problem, Journal of Computer Sciences and Applications, 3, 33-39, 2015.

[22] B. Tobias and K. Walter, An improved deterministic local search algorithm for 3-SAT, Theoretical Computer Science 329, 303-313, 2004.

[23] D. Vilhelm, J. Peter, \& W. Magnus, Counting models for 2SAT and 3SAT formulae. Theoretical Computer Science, 332 (1), 265-291, 2005.

[24] J. Gu, Local Search for Satisfiability (SAT) Problem, IEEE Transactions on Systems, Man and Cybernetics, vol. 23 pp. 1108-1129, 1993.

[25] N. Siddique, H. Adeli, Computational Intelligence Synergies of Fuzzy Logic, Neural Network and Evolutionary Computing. United Kingdom: John Wiley and Sons, 2013.

[26] B. Sebastian, H. Pascal and H. Steffen, Connectionist model generation: A first-order approach, Neurocomputing, 71(13), 2420-2432, 2008.

[27] S. Sathasivam, Upgrading Logic Programming in Hopfield Network, Sains M alay siana, 39, 115-118, 2010.

\section{Authors' Profiles}

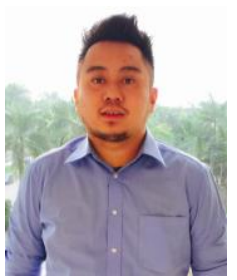

Mohd Asyraf Mansor was born in Sarawak, Malaysia in 1990. He obtain ed his MSc (2014) and BSc(Ed) (2013) from Universiti Sains Malaysia. He is currently pursuing Ph.D degree at School of Mathematical Science, Universiti Sains Malaysia. His current research interests include evolutionary algorithm, satisfiability problem, neural networks, logic programming and heuristic method.

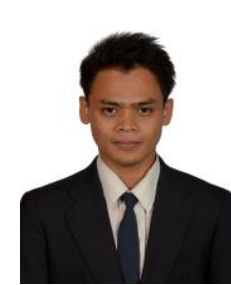

Mohd Shareduwan bin M. Kasihmuddin received his MSc (2014) and $\mathrm{BSc}(\mathrm{Ed})$ (2013) from Universiti Sains Malaysia. $\mathrm{He}$ is currently pursuing Ph.D degree with School of Mathematical Science, Universiti Sains Malaysia Penang Malaysia. His current research interests include neuro-heuristic method, constrained optimization, neural network and logic programming.

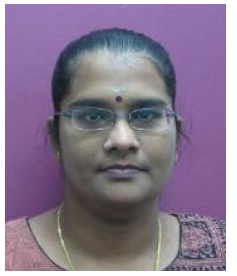

Saratha Sathasivam is an Associate Professor in the School of Mathematical Sciences, Universiti Sains Malaysia. She received her $\mathrm{MSc}$ and $\mathrm{BSc}(\mathrm{Ed})$ from Universiti Sains Malaysia. She received her $\mathrm{Ph} . \mathrm{D}$ at Universiti Malaya, Malaysia. Her current research interest are neural networks, agent based modeling and constrained optimization problem.
How to cite this paper: Mohd. Asyraf Mansor, Mohd Shareduwan M. Kasihmuddin, Saratha Sathasivam, "Enhanced Hopfield Network for Pattern Satisfiability Optimization", International Journal of Intelligent Systems and Applications (IJISA), Vol.8, No.11, pp.27-33, 2016. DOI: 10.5815/ijisa.2016.11.04 